\title{
THE PATHOGENESIS OF LEPROSY
}

\author{
An Experimental Approach \\ G. Weddell and Elisabeth Palmer, \\ Department of Human Anatomy, Oxford.
}

Current views on the pathogenesis of leprosy lead one to suppose that it would be comparatively easy to find acid-fast organisms passing through the epidermis into the skin, particularly in babies in areas where there is repeated, prolonged and intimate contact with the skin of a lepromatous mother. These are the areas which are quite commonly the seat of lesions in the child in later years. However, no one has been able to do this although some authors have maintained that disintegrating bacilli can be found in the form of acid-fast granules in the cells of the superficial layers of the dermis. Be this as it may, no whole mycobacteria have been seen in the skin from contacts, except in their nerves at a stage when they must be regarded as having definitely contracted the disease. It might be argued that the chances of finding organisms in the epidermis are slim but that if relatively large biopsies were homogenized and centrifuged the organisms, which easily resist such procedures, would be concentrated and thus more readily found. Success has been claimed for this technique but we have not been able to find organisms in the skin of contacts by this means, although we have tried many times. Not all the contacts examined were inmates of leprosaria, however.

It is generally accepted that in every patient in whom a diagnosis of leprosy can be established, $M$. leprae are to be seen associated with cutaneous nerves. The problem, as it presented itself to us, was to determine how they reached them. A necessary step and the one we decided to tackle first was the determination of the particular elements in sensory peripheral nerves which were capable of ingesting particulate matter, including mycobacteria. Since the progress of treatment in leprosy is customarily checked from periodical skin biopsies we decided to inject carbon particles into the skin of patients with lepromatous leprosy 5-7 days before such biopsies were taken. We were considerably surprised to find that carbon particles were to be found in large numbers in Schwann cells related to degenerating axons in the cutaneous nerve bundles. We selected cases with the lepromatous form of the disease, for it is known that more cutaneous nerves than usual are passing through a degenerative and regenerative cycles. Clearly, the next step was to analyse the nature of this process and in the early stages this could be done only by using animals for our experiments.

We were fortunate at this point to be able to join forces with Dr. R. J. W. REES and together we carried out a series of controlled 
experiments in which either carbon particles, or $M$. lepraemurium were injected into the sciatic nerves of rats. Healthy animals and others already infected with murine leprosy were used, the particles being injected into crushed nerves or between the stumps of divided nerves. These experiments showed that Schwann cells associated with degenerated nerve fibres behave as macrophages, showing phagocytic activity. They will even ingest $M$. lepraemurium which is not normally associated with nerve fibres in murine leprosy. This is particularly evident in the case of crushed nerves where there are no extraneural macrophages available. Fewer organisms were ingested by Schwann cells in healthy rats than in rats with murine leprosy, but never as freely as carbon particles. The next step was to determine the behaviour of Schwann cells of human sensory cutaneous nerves toward similar forms of particulate matter. To this end small fasciculi from the radial nerves at both wrists were excised and either heat-treated $M$. leprae or $M$. lepraemurium were injected on one side and carbon particles on the opposite side. Fortunately heat-treated $M$. leprae have a characteristic morphology which distinguishes them from the patient's own bacilli.

So far we have only had four volunteers on which we have carried out this kind of experiment but in each case we obtained an unequivocal result: following the injection of $M$. lepraemurium there was an inflammatory response and no organisms were seen in Schwann cells, but they were found in macrophages in the connective tissue sheaths of the nerve bundles. By contrast, $M$. leprae were found in large numbers in Schwann cells which, although only 5 days had elapsed since the original operation, were in a state of heightened activity comparable to that seen normally only 7 days after nerve section. In other words, if it is permissible to generalize from two sets of observations in four patients, certain Schwann cells in degenerating nerves are actively 'attracted' towards killed $M$. leprae. This conclusion was reinforced by another observation in which a segment of the radial nerve serving perfectly normal looking skin, free of sensory impairment, in a patient with a single lesion on the contralateral side, was removed. This was intended to serve as a normal human sensory nerve specimen for testing a new staining method for nerves for examination under the light microscope and comparison with its appearance under the electronmicroscope. We were greatly surprised to find one small bundle within the fasciculus undergoing degenerative changes comparable with those seen in the cutaneous nerve of patients with reactional tuberculoid leprosy, which included fragmented organisms. The findings under the light microscope paralleled those seen under the electronmicroscope but, although a prolonged search was made for mycobacteria in the affected bundle, nothing but acid-fast 'dust' was ever encountered under the light microscope. However, when the intact portion of 
this nerve bundle was examined without expecting any mycobacteria, two viable organisms came to light lying in healthy Schwann cells related to healthy nerve fibres. So far no such organisms have been found under the electronmicroscope but there are still many sections to be examined. It remains to add that no mycobacteria were seen in the skin biopsy from the single patch on the opposite side, the histological picture being typical of tuberculoid leprosy.

Because of the possible significance of these findings we carefully surveyed the literature and have come across some papers reporting the presence of $M$. leprae in the Schwann cells of nerve trunks serving maculo-anaesthetic lesions in patients having a self-limiting form of the disease, who died from causes unconnected with leprosy. These confirmed and extended our own findings. It is also accepted by many authorities that $M$. leprae can be found in the Schwann cells in many nerve trunks of patients with single self-limiting maculo-anaesthetic patches which are quite unrelated to the lesion. Organisms are also present in the affected nerves in the polyneuritic form of leprosy and in some of these cases no skin lesions whatever can be found. Thus our experimental observations, coupled with the reports in the literature, certainly lend no support to the view that inunction is the chief method by which the organism enters the body.

On this point it is necessary to describe some observations on human sensory skin nerves which may appear to be unrelated to leprosy. Our work connected with cutaneous sensibility necessitated the development of improved neurohistological techniques. As the result we can now say with certainty that there is a small but constant turn-over, i.e. degeneration followed by regeneration to be seen in all sensory nerves of the body. The changes are more obvious in those parts exposed to trauma and in the later decades of life.

We have also demonstrated that during Wallerian degeneration the macrophages which remove neural debris stem from within the perineural sheath. Many of them can be seen developing from Schwann cells related to non-myelinated axons.

It is of course premature to argue from this series of preliminary observations that $M$. leprae do not enter the body by inunction through the skin, but they strongly suggest that the evidence for this belief should be re-examined most carefully. Indeed, if our histopathological observations can be substantiated then it must be assumed that leprosy is potentially a highly infectious disease and basically in the same category as tuberculosis.

If we take our experimental observations at their face value then we are compelled to argue as follows: $M$. leprae invade the body by the bloodstream, by inoculation through the skin and, although we have no evidence for this, through the lungs and the intestinal walls. Certainly, inoculation through the skin must occur for cuts, pricks, 
insect bites, etc., are unavoidable in areas where the disease is endemic. Once in the bloodstream the organisms are carried to their target organ, the Schwann cells of the sensory nerves, in which they lie protected by the basement membrane (this is only seen by electronmicroscopy) and multiply. The rate of multiplication is known to be very slow, for proven incubation period are seldom, if ever, less than two years. As in other mycobacterial diseases what follows depends upon: (1) the severity of the infection; (2) the natural immunity of the patient. Sooner or later, most sensory nerve Schwann cells will be involved in the process of Wallerian degeneration, which, as has been explained above, is now known to be a regular event. Those Schwann cells which lose their protective basement membrane and assume the role of phagocytes proceed to remove both neural debris and micro-organisms from the Schwann cells related to the degenerating nerves. If the number of organisms they contain is few then we can assume that the phagocytes will be able to digest them and dispose of them and thereby acquire further anti-body, if natural immunity is present. This will presumably result in the subject becoming lepromin positive and they will exhibit no lesions. In such cases further invasions will be promptly dealt with and even if invasion occurs the organisms will be unable to multiply.

If, however, the original infection is more severe, a larger degree of immunity will have been acquired. Thus, when the number of organisms is large enough to break through the protective basement membranes, there will be an explosive antibody-antigen reaction, a maculo-anaesthetic patch will appear and the disease will become self-limiting. Although maculo-anaesthetic lesions usually lead to a self-limiting form, this may not always be the case. In the "African macule' a tuberculoid lesion is the first to appear and the patient is lepromin positive. Later, however, further lesions appear and the patient may even, in time, present with a lepromatous lesion and is found to be lepromin negative. This series of events can, if our assumptions prove correct, be explained on the basis of a low natural immunity coupled with repeated and massive doses of infection. The polyneuritic form of the disease, which is the most distressing of all, probably results from multiple severe infections in subjects with low natural immunity. Finally, the lepromatous form can be regarded as a result of massive infection in patients who have no natural immunity.

In different parts of the world and among peoples of different genetic stock the forms of the disease vary considerably. It seems that, genetically speaking, a greater resistance is inherited by some races than others.

There would thus appear to be three factors concerned in the pathogenesis of leprosy; (1) the severity of the infection; (2) natural 
immunity, probably genetic in origin; (3) racial immunity, certainly genetic in origin.

This view brings leprosy into line with other mycobacterial diseases. If we are right, then it must be the long incubation period which has given rise to the suggestion that $M$. leprae has a low degree of infectivity. Indeed, it is difficult to understand how certain patients develop the disease often many years after living in countries where it was endemic, unless the organism is regarded as potentially very infectious. On the basis of our work such cases might well be the result of a sudden increase in neural turn-over and the release of a large number of organisms. The site of the lesion in leprosy, we would like to emphasize, is in our view no indication of the portal of entry but rather an expression of damage to the nerves in the skin, or at any point between the skin and spinal cord.

We should like to make it quite clear, that our description of the pathogenesis of leprosy must not be regarded as already proven. It is based as yet on a few experimental observations which must be validated statistically.

It seemed to us that leprologists would wish to know of our findings at this stage because they suggest so strongly that education in personal and public hygiene is just as important in the case of leprosy as it is in tuberculosis. Most workers in the field have already assumed this; all we have tried to do is to supply them with some ammunition, not necessarily for use in action but for thought.

As laboratory workers we are only anxious to help, not to issue edicts. We are well aware that in the long run it is the leprologist only who can solve the problem of the eradication of leprosy.

\section{Summary}

The authors describe certain of their findings in the histology of the skin and nerves which cause them to doubt whether inunction through the skin is as important as was previously thought in the pathogenesis of leprosy. They also call for attention to three factors (1) the severity of the infection; (2) natural immunity, probably genetic in origin; (3) racial immunity, certainly genetic in origin, and call for statistical and other research. 